\title{
The effect of volcanic aerosols on ultraviolet radiation in Antarctica
}

\author{
Steven R. Tsitas and Yuk L. Yung \\ Division of Geological and Planetary Sciences, California Institute of Technology, Pasadena
}

\begin{abstract}
Volcanic eruptions can inject large amounts of aerosol into the atmosphere, and, at large solar zenith angles, scattering by these aerosols can actually increase the flux of UV-B (290-320 $\mathrm{nm}$ ) radiation reaching the surface. This is surprising since aerosols increase the reflection of sunlight to space. As previous explanations of this phenomenon are heuristic and incomplete, we first provide a rigorous and complete explanation of how this surprising effect occurs. This phenomenon makes Antarctica during spring the most susceptible place on Earth to the scattering effect of volcanic aerosols, due to the combined effect of the spring ozone hole and the large solar zenith angles characteristic of this time of year. We show that an aerosol layer lying above Antarctica during spring will decrease the integrated daily dose of biologically weighted irradiance, weighted by the erythema action spectrum, by only up to $5 \%$. Hence the effects of any significant destruction of ozone induced by volcanic aerosols will not be offset by aerosol scattering. Thus after a volcanic eruption, life in Antarctica during spring will suffer the combined effects of the spring ozone hole and ozone destruction induced by volcanic aerosols, with the latter effect only slightly offset by aerosol scattering.
\end{abstract}

\section{Introduction}

Recent numerical solutions of the equation of radiative transfer [Michelangeli et al., 1989; Michelangeli et al., 1992; Tsay and Stamnes, 1992] indicate that, at large solar zenith angles, scattering by a stratospheric aerosol layer can increase the flux of ultraviolet light reaching the surface. An explanation of this phenomenon, which has not yet been observed, has been offered by Davies [1993]. Basically, at large solar zenith angles, scattering from a stratospheric aerosol layer provides a shortcut for ultraviolet light, which is strongly absorbed by ozone, to reach the ground. This scattered light suffers much less attenuation than the direct solar beam, which travels a long slant path through the atmosphere. Hence there is an increase in flux at the ground, even though some of the scattered light escapes to space, increasing that flux also. Davies uses a model of the atmosphere which treats the aerosol as a single scattering layer, but neglects Rayleigh scattering by the atmosphere in any approximation, to demonstrate that this mechanism can lead to an enhancement of surface flux. However, at ultraviolet wavelengths the atmosphere is strongly Rayleigh scattering (the Rayleigh scattering optical depth in the zenith direction is 1 at $305 \mathrm{~nm}$ ), and so it has not been shown if the mechanism proposed by Davies is the one responsible for surface flux enhancement in a realistic, Rayleigh scattering atmosphere.

Copyright 1996 by the American Geophysical Union.

Paper number 95GL03592

0094-8534/96/95GL-03592\$03.00

\section{Explanation of the Mechanism of Enhancement}

To investigate this phenomenon, we use a 30 -stream radiative transfer model of the atmosphere that is based on the numerical solution of the equation of radiative transfer as formulated by Michelangeli et al. [1992], which is suitable for treating an aerosol layer. Our numerical model includes Rayleigh scattering by air, absorption by ozone, aerosol scattering and absorption and assumes a Lambert surface. The attenuation of the direct solar beam is treated using spherical geometry, while the multiple scattering is calculated for a plane-parallel atmosphere [Froidevaux et al., 1985]. Rayleigh scattering and ozone absorption cross sections, and the solar flux incident upon the top of the atmosphere, are taken from the World Meteorological Organization [1986] report. Volcanic aerosols are assumed to scatter with the Henyey-Greenstein phase function [Henyey and Greenstein, 1941], with an asymmetry parameter of 0.75 and single scattering albedo of 0.99 [Vogelmann et al., 1992].

We validated our model by calculating the diffuse reflection and transmission functions for light incident upon a plane-parallel slab of scatterers, and comparing the results with the tables published by van de Hulst [1980], for 120 widely varied sets of values of the relevant parameters. The 120 calculated diffuse transmission functions differ from van de Hulst's tabulated values by an average of $0.13 \%$ and a maximum of $1.5 \%$. Corresponding values for the 120 diffuse reflection functions are $0.33 \%$ and $5.6 \%$, respectively, with all the differences greater than $0.60 \%$ occurring when the incident beam is normal to the slab, and the diffuse reflection function is calculated in the direction back along the incident beam. Since we only use our model to calculate the flux transmitted to the surface at large solar zenith angles, the results presented in this paper have a fractional error of less than $1-2 \%$.

We have used our numerical model to calculate the factor, $E$, by which the surface flux is enhanced by a volcanic aerosol layer, assumed to spread uniformly from $25-26 \mathrm{~km}$ altitude [DeLuisi et al., 1983] with an optical depth of 0.4 [Valero and Pilewskie, 1992]. This factor, calculated for a solar zenith angle of $80^{\circ}$, is shown in the second column of Table 1. A surface albedo of 0.05 was used in the calculations, and we assumed that the atmosphere is the U.S. Standard Atmosphere [1976], with the amount of ozone scaled to $200 \mathrm{DU}$. The first column of Table 1 lists the approximate center wavelengths of the wavelength intervals from the World Meteorological Organization [1986] report. $\tau_{\mathrm{O}_{3}}$ is the optical depth of the atmosphere, in the zenith direction, due to ozone absorption. The fourth and fifth columns show the percentage of downward travelling light that is due to direct (unscattered) sunlight at $26 \mathrm{~km}$ altitude and at the surface, respectively, in the clear atmosphere. The last column gives the enhancement factor, $\mathrm{E}$, for surface irradiance calculated using Davies' [1993] model (the fraction of $\tau_{\mathrm{O}_{3}}$ beneath the aerosol layer is 0.672 , independent of wavelength).

We do not make the comparison with Davies' model to show its inaccuracy, for Davies' model is obviously intended to be 
Table 1. Enhancement of Flux by a Stratospheric Aerosol Layer

\begin{tabular}{llllll}
\hline $\begin{array}{c}\text { Wavelength } \\
\text { (nm) }\end{array}$ & $\mathrm{E}$ & $\tau_{\mathrm{O}_{3}}$ & $\begin{array}{c}\text { \% Direct } \\
(26 \mathrm{~km})\end{array}$ & $\begin{array}{l}\text { \% Direct } \\
\text { (surface) }\end{array}$ & $\begin{array}{c}\text { E-Davies' } \\
\text { model }\end{array}$ \\
\hline 292 & 0.919 & 5.6 & 14 & $9.4 \times 10^{-9}$ & $1.5 \times 10^{6}$ \\
296 & 1.22 & 3.1 & 65 & $1.6 \times 10^{-4}$ & $8.8 \times 10^{2}$ \\
301 & 2.22 & 1.7 & 86 & $4.1 \times 10^{-2}$ & $1.6 \times 10^{1}$ \\
305 & 2.22 & 0.89 & 92 & 0.47 & 2.0 \\
310 & 1.56 & 0.47 & 93 & 1.2 & 0.76 \\
315 & 1.18 & 0.23 & 94 & 1.9 & 0.48 \\
320 & 1.01 & 0.11 & 94 & 2.7 & 0.39 \\
325 & 0.939 & 0.050 & 95 & 3.5 & 0.36 \\
\hline
\end{tabular}

qualitative. But its purpose is to demonstrate the correctness of his intuitive explanation for the mechanism responsible for the enhancement in a realistic atmosphere. The point we wish to make by the comparison is that Davies' model fails to reproduce even qualitatively the decrease in enhancement predicted at the shortest ultraviolet wavelengths. Also, since Davies' model does not include Rayleigh scattering by the atmosphere, it assumes that in the clear atmosphere the radiation at the surface is just the direct radiation, attenuated by ozone absorption. The penultimate column of Table 1 shows that this is not a valid approximation throughout the wavelength range of the enhancement. For these two reasons we claim that Davies' explanation for the enhancement has not been shown to apply to a realistic, Rayleigh scattering atmosphere.

To rigorously demonstrate the mechanism responsible for enhancement in a realistic, Rayleigh scattering atmosphere, we plot the direct, diffuse and total irradiance as a function of altitude both with and without an aerosol layer. Two diffuse irradiances are plotted. One (dashed curve) is the cosine weighted integration of the diffuse intensity over the upward hemisphere, representing the flux of diffuse light travelling up through the atmosphere. The other (dotted-dashed curve) is the cosine weighted integration of the diffuse intensity over the downward hemisphere, representing

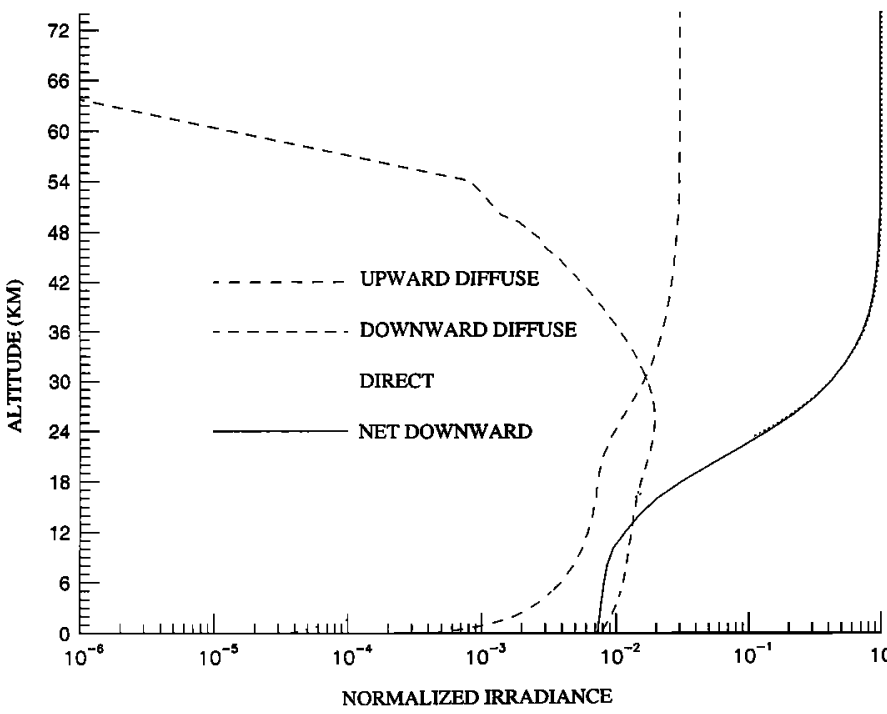

Figure 1. The upward diffuse, downward diffuse, direct and net downward irradiance, normalized to the solar irradiance incident perpendicularly upon the top of the atmosphere, in the wavelength range $303.03-307.7 \mathrm{~nm}$ as a function of altitude in an aerosol-free U.S. Standard Atmosphere [1976], weighted to 200 DU. Net downward $=$ direct + downdiffuse - updiffuse. The solar zenith angle is $80^{\circ}$.

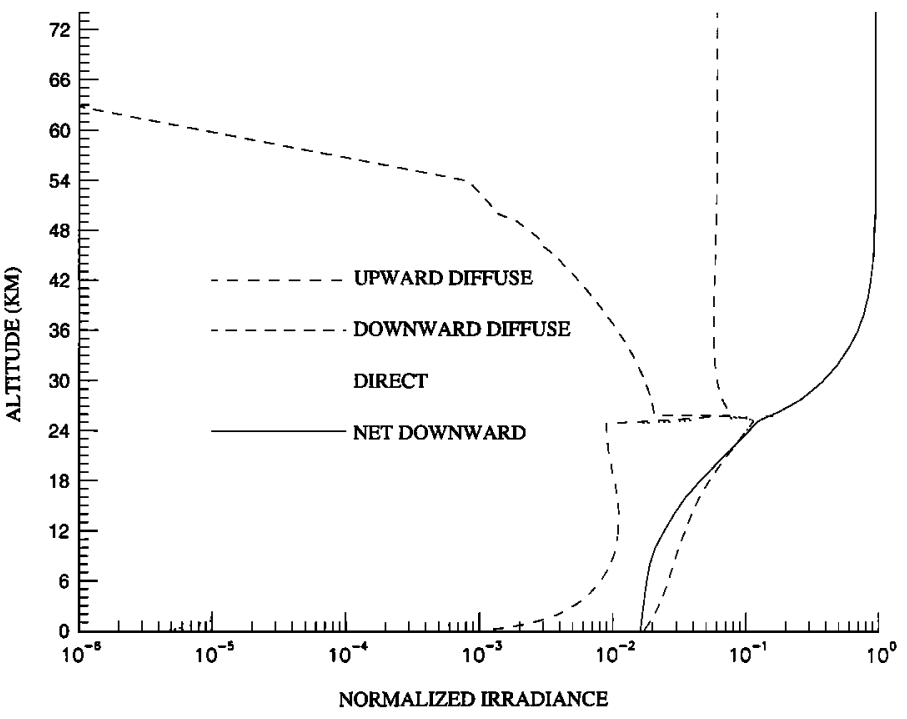

Figure 2. With the introduction of an aerosol layer of optical depth 0.4 , extending from $25-26 \mathrm{~km}$ altitude, the upward diffuse, downward diffuse, direct and net downward irradiance become the functions of altitude shown. The aerosol layer increases the flux at the surface by more than a factor of 2 by converting direct sunlight into diffuse (scattered) light.

the flux of diffuse light travelling down through the atmosphere. The net downward irradiance is the direct irradiance plus the diffuse irradiance integrated over the downward hemisphere minus the diffuse irradiance integrated over the upward hemisphere. We consider the wavelength range $303.0-307.7 \mathrm{~nm}$, for which the surface flux enhancement factor is 2.22 , as given in Table 1. The upward diffuse, downward diffuse, direct and net downward irradiance as a function of altitude in the aerosol free atmosphere are shown in Figure 1. The introduction of an aerosol layer of optical depth 0.4 , extending from $25-26 \mathrm{~km}$ altitude, causes the upward diffuse, downward diffuse, direct and net downward irradiance to become the functions of altitude shown in Figure 2. These irradiances are normalized to the solar irradiance incident perpendicularly upon the top of the atmosphere.

By comparing the Figures we see that the aerosol layer increases the downward diffuse irradiance at $25 \mathrm{~km}$ altitude by a factor of 5 . This is due to the conversion of direct sunlight into diffuse (scattered) light by the aerosol. Since the total irradiance at the surface is overwhelmingly due to downward diffuse light, the decrease in direct sunlight at the surface due to the aerosol layer is more than compensated for by the increase in downward diffuse light. Hence, as long as the radiation at the altitude of the top of the aerosol layer is primarily direct, enhancement of surface flux occurs by the mechanism described by Davies [1993]. However, Davies' model fails to predict the decrease in enhancement seen at the shortest ultraviolet wavelengths, because such wavelengths are both strongly Rayleigh scattered and ozone absorbed by the atmosphere. Therefore, little of the radiation is $10^{\circ}$ direct sunlight by the altitude of the top of the aerosol layer (most is scattered light), as can be seen from the fourth column of Table 1. Hence scattering by the aerosol layer provides no shortcut to the ground, and, because of backscatter to space, the flux at the surface actually decreases.

We extended Davies' model to include Rayleigh scattering by the atmosphere in the single scattering approximation, assuming the same type of atmosphere and aerosol as was considered in calculating the results presented in Table 1 . In this case no 
enhancement of surface flux occurs in any of the wavelength intervals considered in Table 1 . This is because a single scattering calculation always underestimates the diffuse light reaching the surface; since the enhancement is due to an increase in diffuse light, no enhancement will be predicted if the model significantly underestimates the transmission of diffuse light to the surface.

\section{Biological Significance}

Large solar zenith angles and the ozone hole make Antarctica during spring the most susceptible place on Earth to the scattering effect of volcanic aerosols. Accordingly, we have used our numerical model to calculate the effect of a volcanic aerosol layer on indigenous life in Antarctica during spring. For a representative day in Antarctica during the spring ozone hole (October 7), and for a representative region near the edge of the pack ice where most indigenous life is located $\left(70^{\circ} \mathrm{S}\right)$, we calculate the flux, from $290-420 \mathrm{~nm}$, transmitted to the surface both with and without a stratospheric aerosol layer. The baseline atmosphere is the U.S. Standard Atmosphere [1976], with the amount of ozone scaled to $200 \mathrm{DU}$ to represent the effect of the ozone hole (about 35\% below normal). We assume that the lower boundary of the atmosphere is the ocean, with an albedo of 0.05 [Doda and Green, 1980; Blumthaler and Ambach, 1988], assumed constant from $290-420 \mathrm{~nm}$. The flux is calculated for 10 solar zenith angles between sunrise and noon (solar zenith angle = $64.5^{\circ}$ ).

The spectral irradiance (flux per unit wavelength interval) at each solar zenith angle is convolved with an action spectrum. The integrated daily dose of radiation [Lubin et al., 1992] is approximated by the sum of the 10 convolutions weighted by the length of time each solar zenith angle is assumed to exist for. The convolution is calculated from $290-420 \mathrm{~nm}$ in $0.1 \mathrm{~nm}$ steps. It is necessary to calculate the change in the integrated daily dose of radiation, rather than just the change in the mid-day dose, because about half the daily dose of radiation is acquired at times other than mid-day, and the enhancement is much larger at these solar zenith angles than at mid-day.

The action spectrum is the relative effectiveness of different wavelengths in causing a biological response. We use the action spectrum for DNA [Setlow, 1974] for reference. This action spectrum is probably not representative of the effect on any biological organism. However, many previous studies of the transmission of ultraviolet radiation through the atmosphere use this action spectrum, and so we have included it for comparison purposes only. The action spectra we have chosen to be representative of the biological response of actual organisms are the phytoplankton action spectrum [Mitchell, 1990; Lubin et al., 1992], and the erythema action spectrum [Diffey, 1987]. The choice of a phytoplankton action spectrum is obvious, since the oceans off Antarctica contain abundant phytoplankton. We chose the erythema action spectrum as a generic representation of the possible biological response of the eyes of birds and the eyes and skin of marine mammals which may come close to the ocean surface, or out of the water entirely onto the ice. These three action spectra differ mainly in the strength of the UV-A (320-420 $\mathrm{nm}$ ) "tail" of the spectra relative to the weighting of the action spectra in the UV-B (290-320 nm). The DNA action spectrum has a negligible UV-A tail, the erythema UV-A tail is more prominent relative to its UV-B weighting, and the phytoplankton action spectrum has the strongest UV-A tail relative to UV-B weighting. Since the flux of UV-B is in general increased by the aerosol layer, while the flux of UV-A is decreased by the aerosol layer, the change in the integrated daily dose of radiation depends very strongly on which action spectrum is used to weight the spectral irradiance. The action spectrum with the weakest UV-A tail relative to UV-B weighting (DNA) will produce the most pronounced increases in the integrated daily dose of biologically weighted irradiance. By contrast, the action spectrum with the strongest relative UV-A tail (phytoplankton) is expected to show a decrease in the integrated daily dose of biologically weighted irradiance, because the decrease in UV-A, relatively strongly weighted by the action spectrum, outweighs the increase in UV-B.

The change in the integrated daily dose of biologically weighted irradiance, caused by a stratospheric aerosol layer being introduced into the baseline atmosphere, is given in Table 2. The columns labelled "PP", "ERY" and "DNA" refer to weighting by the phytoplankton, erythema and DNA action spectra, respectively. The range of aerosol layer optical depths $(\tau)$ bounds values observed after volcanic eruptions [Hofmann, 1987; Valero and Pilewskie, 1992]. The aerosol is assumed to be uniformly distributed within 2 altitude ranges representative of observations made after eruptions, 12-24 km [McCormick et al., 1984] and 25 $26 \mathrm{~km}$ [DeLuisi et al., 1983], the latter altitude range observed at Mauna Loa about 1 week after the El Chichon eruption. This layer spread vertically with time [DeLuisi et al., 1983], so for a similar layer to exist over Antarctica, the eruption would have to occur in or near to Antarctica. Observations of the aerosol cloud injected into the stratosphere by the Mt. Pinatubo eruption [Osborn et al., 1995] indicate that this cloud spread from a narrow initial height range of $22-24 \mathrm{~km}$, observed 2 months after the eruption, to a much broader and lower height range of $16-24 \mathrm{~km}$, observed 12 months after the eruption. The observations indicate that the aerosol cloud descended and spread out at a roughly constant rate during this 10 month period. Hence we consider the narrow aerosol range considered in this paper to only be valid for one to two months following an eruption, requiring a volcanic eruption in Antarctica. Since volcanic aerosols may induce ozone destruction [Vogelmann et al., 1992], we repeated the calculations for the 12-24 km layer assuming an $8 \%$ ozone column depletion (to $184 \mathrm{DU}$ ), due to uniform depletion within the layer. This is representative of ozone column depletions observed at some locations after the El Chichon eruption [Bojkov, 1987]. There is not enough ozone between 25 and $26 \mathrm{~km}$ altitude for an aerosol induced ozone depletion there to be significant.

For reference, the increase in the integrated daily dose of biologically weighted irradiance, weighted by the DNA action spectrum (BWI-DNA), due to an aerosol layer extending from $12-24 \mathrm{~km}$, with an $8 \%$ ozone column depletion (to $184 \mathrm{DU}$ ), is given in Table 2 and may be compared directly with the results of Vogelmann et al. [1992]. Their results were calculated for midlatitudes, where scattering by an aerosol layer decreases the flux of ultraviolet light at the surface, offsetting the effects of aerosol induced ozone destruction [Vogelmann et al., 1992]. Vogelmann

Table 2. Percentage Change in the Integrated Daily Dose of Biologically Weighted Irradiance (Positive = Increase), Due to an Aerosol Layer Lying Above Antarctica

\begin{tabular}{cccccccccc}
\hline & \multicolumn{1}{c}{$12-24 \mathrm{~km}(200 \mathrm{DU})$} & \multicolumn{1}{c}{ 12-24 km (184 DU) } & \multicolumn{2}{c}{$25-26 \mathrm{~km}(200 \mathrm{DU})$} \\
$\tau$ & PP & ERY & DNA & PP & ERY & DNA & PP & ERY & DNA \\
\hline 0.0 & 0 & 0 & 0 & 1.8 & 11 & 19 & 0 & 0 & 0 \\
0.1 & -3.0 & -1.5 & 0.0 & -1.2 & 9.4 & 20 & -2.4 & 0.8 & 4.8 \\
0.2 & -5.8 & -2.7 & 0.3 & -4.0 & 9.0 & 21 & -4.7 & 1.7 & 9.3 \\
0.3 & -8.4 & -4.9 & -0.8 & -6.5 & 7.9 & 22 & -6.7 & 2.6 & 13 \\
0.4 & -11 & -5.1 & 0.4 & -8.9 & 4.5 & 18 & -8.6 & 3.4 & 17 \\
\hline
\end{tabular}


et al. predict increases of approximately $8-15 \%$ for $\tau=0.4-0.1$, which if applied to Antarctica would underestimate the increase in BWI-DNA there by up to a factor of 2 .

Volcanic aerosols must induce significant ozone destruction in order for there to be an increase in BWI-DNA at mid-latitudes [Vogelmann et al., 1992]. However, the Mt Pinatubo eruption caused zonal-scale ozone depletions of only $2-4 \%$ within the aerosol layer, so that scattering by the aerosols effectively offset the ozone destruction [Vogelmann et al., 1992]. By contrast, the last column in Table 2 shows that a narrow aerosol layer lying above Antarctica during spring can cause large (up to 17\%) increases in BWI-DNA, even if the aerosols do not induce any ozone destruction.

Fortunately, however, the effect of a volcanic aerosol layer on the integrated daily dose of biologically weighted irradiance is much less severe when the phytoplankton and erythema action spectra are used. These action spectra are expected to more realistically mimic the biological response of life in Antarctica to ultraviolet radiation. Phytoplankton are actually better off with an aerosol layer overhead, because while the flux of UV-B radiation is increased, the flux of UV-A radiation is decreased, and the relatively strong UV-A weighting of the phytoplankton action spectrum leads to an overall decrease in biological response, even with an $8 \%$ reduction of ozone due to aerosol induced ozone destruction. However, the third column of Table 2 shows that when the irradiance is weighted with the erythema action spectrum, the integrated daily dose of biologically weighted radiation (BWI-ERY) decreases by only up to $5 \%$ with the introduction of an aerosol layer. This is not enough to offset the effects of an $8 \%$ ozone depletion due to ozone destruction within the aerosol layer (sixth column of Table 2). Larger aerosol induced ozone depletions will be offset by only up to the same $5 \%$, so it is possible for volcanic aerosols to cause significant increases in BWI-ERY in Antarctica during spring. These increases would be in addition to the effects of the higher than normal levels of ultraviolet radiation in Antarctica caused by the yearly spring ozone hole.

Acknowledgments. This research was supported by grants from the National Aeronautics and Space Administration, through grant number NAGW-413, and the Environmental Protection Agency, through grant number CR818670-01-0.

\section{References}

Blumthaler, M., and W. Ambach, Solar UVB-albedo of various surfaces, Photochem. Photobiol., 48, 85-88, 1988.

Bojkov, R. D., The 1983 and 1985 anomalies in ozone distribution in perspective, Mon. Weath. Rev., 115, 2187-2201, 1987.

Davies, R., Increased transmission of ultraviolet radiation to the surface due to stratospheric scattering, J. Geophys. Res., 98, 7251-7253, 1993.

DeLuisi, J. J., E. G. Dutton, K. L. Coulson, T. E. DeFoor, and B. G. Mendonca, On some radiative features of the El Chichon volcanic stratospheric dust cloud and a cloud of unknown origin observed at Mauna Loa, J. Geophys. Res., 88, 6769-6772, 1983.

Diffey, B. L., A comparison of dosimeters used for solar ultraviolet radiometry, Photochem. Photobiol., 46, 55-60, 1987.
Doda, D. D., and A. E. S. Green, Surface reflectance measurements in the UV from an airborne platform. Part 1, Applied Optics, 19, 2140-2145, 1980.

Froidevaux, L., M. Allen, and Y. L.Yung, A critical analysis of CLO and 03 in the mid-latitude stratosphere, J. Geophys. Res., 90, 12,999$13,029,1985$.

Henyey, L. C., and J. L. Greenstein, Diffuse radiation in the galaxy, Astrophys. J., 93, 70-83, 1941.

Hofmann, D. J., Perturbations to the global atmosphere associated with the El Chichon volcanic eruption of 1982, Rev. Geophys., 25, 743-759, 1987.

Lubin, D., B. G. Mitchell, J. E. Frederick, A. D. Alberts, C. R. Booth, T. Lucas, and D. Neuschuler, A contribution toward understanding the biospherical significance of Antarctic ozone depletion, $J$. Geophys. Res., 97, 7817-7828, 1992.

McCormick, M. P., T. J. Swissler, W. H. Fuller, W. H. Hunt, and M. T. Osborn, Airborne and ground-based lidar measurements of the $\mathrm{El}$ Chichon stratospheric aerosol from $90^{\circ} \mathrm{N}$ to $56^{\circ} \mathrm{S}$, Geofis. int., 23, 187-221, 1984

Michelangeli, D. V., M. Allen, and Y. L. Yung, El Chichon volcanic aerosols: impact of radiative, thermal, and chemical perturbations, $J$. Geophys. Res., 94, 18,429-18,443, 1989.

Michelangeli, D. V., M. Allen, Y. L. Yung, R.-L. Shia, D. Crisp, and J. Eluszkiewicz, Enhancement of atmospheric radiation by an aerosol layer, J. Geophys. Res., 97, 865-874, 1992.

Mitchell, B. G., Action spectra of ultraviolet photoinhibition of Antarctic phytoplankton and a model of spectral diffuse attenuation coefficients, in Response of Marine Phytoplankton to Natural Variations in UV-B Flux, edited by G. Mitchell, I. Sobolev, and O. Holm-Hansen, Appendix H, Chemical Manufacturers Association, Washington, D.C., 1990.

Osborn, M. T., R. J. DeCoursey, C. R. Trepte, D. M. Winker, and D. C Woods, Evolution of the Pinatubo volcanic cloud over Hampton, Virginia, Geophys. Res. Lett., 22, 1101-1104, 1995.

Setlow, R. B., The wavelengths in sunlight effective in producing skin cancer: A theoretical analysis, Proc. Natn. Acad. Sci.. USA, 71, 3363$3366,1974$.

Tsay, S. C., and K. Stamnes, Ultraviolet-radiation in the Arctic: the impact of potential ozone depletions and cloud effects, J. Geophys. Res., 97 , 7829-7840, 1992.

U.S. Standard Atmosphere, NOAA-S/T 76-1562, U.S. Government Printing Office, Washington D.C., 1976.

Valero, F. P. J., and P. Pilewskie, Latitudinal survey of spectral optical depths of the Pinatubo volcanic cloud - derived particle sizes, columnar mass loadings, and effects on planetary albedo, Geophys. Res. Lett., 19, 163-166, 1992.

van de Hulst, H. C., Multiple light scattering, volume 2, Academic, San Diego, pp. 412-443, 1980.

Vogelmann, A. M., T. P. Ackerman, and R. P. Turco, Enhancements in biologically effective ultraviolet radiation following volcanic eruptions, Nature, 359, 47-49, 1992.

World Meteorological Organization, Atmospheric Ozone 1985: Assessment of our understanding of the processes controlling its present distribution and change, Rep. 16, chap. 7, pp. 349-392, Global Ozone Research and Monitoring Project, Geneva, 1986.

S. R. Tsitas, Division of Geological and Planetary Sciences 170-25, California Institute of Technology, Pasadena, CA 91125. (e-mail: sit@cco.caltech.edu) (Corresponding Author)

Y. L Yung, Division of Geological and Planetary Sciences 170-25, Califomia Institute of Technology, Pasadena, CA 91125. (e-mail: yly@mercul.gps.caltech.edu)

(Received April 24, 1995; revised September 2, 1995; accepted November 2, 1995) 